\title{
Monitoring p53's pulse
}

\author{
John J Tyson \\ The tumor-suppressor protein p53 mediates the response of mammalian cells to DNA damage. Studies now show that \\ DNA damage elicits quantal pulses of p53, with greater damage eliciting more pulses.
}

Just as the blueprint of a building may be marred by rips or coffee spills, the blueprint of life - DNA - may be damaged by sunlight or noxious chemicals. Most nicks and smudges are innocuous, but some changes to the plan, if not repaired, can have catastrophic consequences for the structural integrity of the building or the viability of the organism ${ }^{1}$. For this reason, master plans are carefully maintained in the architect's office, and DNA is closely monitored in the cell's nucleus. The chief monitor in mammalian cells is a protein called p53, whose job is to respond to DNA damage by inducing repair enzymes and, if the damage is too severe, by mobilizing a program of cell suicide. One might guess that the amount of p53 in a given cell would increase in proportion to the extent of damage, but, on page 147, Lahav et al. ${ }^{2}$ show that the severity of DNA damage is encoded in the number of discrete pulses of constant-amplitude waves of p53. This discovery has far-reaching implications for our understanding of how p53 protects us from the potentially carcinogenic consequences of DNA lesions.

\section{Pulse rate}

Cells contain networks of interacting proteins that process information and compute a response appropriate to the type and strength of the signals received ${ }^{3-5}$. Bacteria swim toward food, eggs mature in response to hormones, retinal cells fire when struck by photons. The signal-response curve is rarely a linear proportion or a saturating hyperbola (like reaction rate as a function of substrate concentration for a Michaelis-Menten enzyme). More often, responses tend to be sigmoidal functions of signals, with abrupt transitions ( $\mathrm{OFF} \leftrightarrow \mathrm{ON}$ ) as signal strength passes back and forth across a threshold. In some cases, the response may be 'hysteretic' (OFF $\rightarrow$ ON at some threshold, and ON $\rightarrow$ OFF at a lower threshold) or even irreversible (OFF $\rightarrow$ ON, never to return).

In the late 1990s, Uri Alon, Arnold Levine and their colleagues set out to measure the signal-response relation between DNA damage and p53 expression. Using antibodies

John J. Tyson is at Virginia Polytechnic Institute and State University, Blacksburg, Virginia 0610406,USA.e-mail:tyson@vt.edu

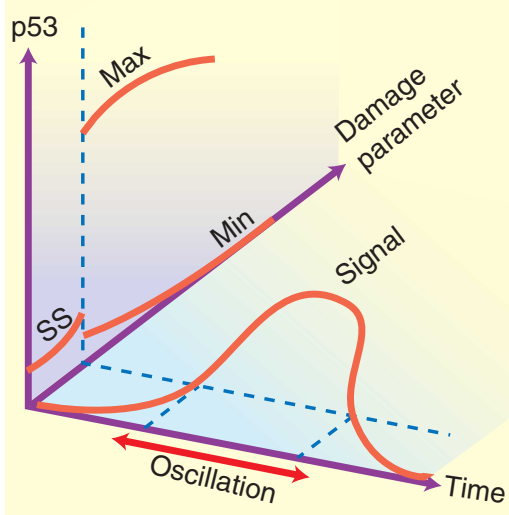

Figure 1 A theoretical account of how p53 oscillations may depend on the extent of DNA damage. For low damage, p53 level persists at a low, steady-state level (SS). For high damage, p53 oscillates between two extreme values (Min and Max). If DNA damage is induced and then repaired (Signal curve), the cell's response will be a finite number of $\mathrm{p} 53$ pulses generated when the 'damage parameter' is above threshold (dashed line).

against p53, they measured average protein level in populations of mouse cells exposed to high doses of ionizing radiation ${ }^{6}$. They saw what resembled a damped oscillation in p53 expression and presented a simple theoretical explanation based on the well-known negative feedback between p53 and Mdm2, a protein required for p53 degradation. (p53 stimulates synthesis of Mdm2, which destroys p53, thus turning off its own production.) This experiment suggests an unexpected oscillatory response, but it is inconclusive because the antibody technique requires pooling proteins from many cells harvested at specific times after irradiation.

Lahav et al. ${ }^{2}$ set out to address what was happening in individual cells using fluorescently labeled versions of $\mathrm{p} 53$ and $\mathrm{Mdm} 2$ that can be visualized and quantified. They found that, in response to ionizing radiation, cells emit p53 in pulses of fixed height and duration. Though genetically identical, cells emit different numbers of pulses (resembling a damped oscillation when averaged over a population of cells), and the mean number of pulses increases with the extent of DNA damage.

\section{Different but the same}

Although these observations are unexpected, there are examples of sustained oscillatory responses in other contexts. In response to pressure, temperature, light and such, sensory nerves emit quantized action potentials, and the strength of the signal is encoded in the frequency and duration of the wave train ${ }^{7}$. Many hormones are secreted in a pulsatile manner, and the second messengers, $\mathrm{Ca}^{2+}$ and cyclic AMP, often have oscillatory responses ${ }^{8}$. The mechanisms for such oscillatory responses are based on the positive and negative feedback loops built into networks of interacting regulatory proteins ${ }^{4,5,8}$. Under certain precise, computable and experimentally verifiable conditions, these loops may generate sustained oscillatory outputs.

In general terms, the connection between signal strength and oscillatory response is captured in a bifurcation diagram (Fig. 1). In the plane spanned by the axes 'p53' and 'damage parameter', the sustained response of the signal transduction network to different levels of damage is plotted. At low damage levels, the response is a low, steady level of p53 expression. At high damage levels, the response is an oscillation of p53 (and Mdm2 and all other proteins involved in the network) between the minimum and maximum levels plotted on the diagram. The switch between steady state and oscillatory responses occurs at a threshold damage level, called the bifurcation point. Figure 1 represents one common type of bifurcation to oscillations of relatively fixed amplitude and frequency.

In response to ionizing radiation, the damage parameter will first increase in time and then decrease, as the damage is repaired. As observed, the output of the control system will be pulses of p53 of fixed amplitude and frequency for as long as the damage parameter is above the bifurcation point. It is presumed that the cell can count the number of pulses, more pulses corresponding to increasingly aggressive responses to the damage. If the damage parameter stays in the oscillatory region for a long time, because the damage is severe or repair mechanisms are faulty, then the resulting, long string of $\mathrm{p} 53$ pulses may be sufficient to initiate apoptosis. 
The connection between oscillations and feedback in cellular regulatory systems goes back to the 1960s and early 1970s, when experimentalists studying glycolytic oscillations, mitotic and circadian rhythms, periodic enzyme synthesis and cyclic AMP oscillations joined forces with theoreticians to speculate on underlying molecular mechanisms that were, in many cases, beyond the power of the biochemical techniques of the day ${ }^{9}$. In the 1980s' frenzy to crack open these black boxes with the tools of molecular genetics, the rapport between experimentalists and theoreticians fell by the wayside. The work of Lahav et al. joins several recent publications that demonstrate a renewed vitality of molecular genetics when combined with sound theoretical and computational analysis of macromolecular regulatory systems $^{10-14}$. This rapprochement promises deeper insights into how a cell tailors its response to internal and external signals.

\footnotetext{
1. Hanahan, D. \& Weinberg, R.A. Cell 100, 57-70 (2000).

2. Lahav, G. et al. Nat. Genet. 36, 147-150 (2004).

3. Bray, D. Nature 376, 307-312 (1995).

4. Ferrell, J.E. Jr \& Xiong, W. Chaos 11, 227-236 (2001).

5. Tyson, J.J., Chen, K.C. \& Novak, B. Curr. Opin. Cell Biol. 15, 221-231 (2003).

6. Lev Bar-Or, R. et al. Proc. Natl. Acad. Sci. USA $\mathbf{9 7}$,
}

11250-11255 (2000)

7. Dayan, P. \& Abbott, L.F. Theoretical Neuroscience: Computational and Mathematical Modeling of Neural Systems (MIT Press, Cambridge, 2001).

8. Goldbeter, A. Biochemical Oscillations and Cellular Rhythms (Cambridge University Press, Cambridge, 1996).

9. Chance, B., Pye, E.K., Ghosh, A.K. \& Hess, B. (eds.) Biological and Biochemical Oscillators (Academic, New York, 1973).

10. Elowitz, M.B. \& Leibler, S. Nature 403, 335-338 (2000).

11. Hoffman, A., Levchenko, A., Scott, M.L. \& Baltimore, D. Science 298, 1241-1245 (2002).

12. Xiong, W. \& Ferrell, J.E. Jr Nature 426, 460-465 (2003).

13. Sha, W. et al. Proc. Natl. Acad. Sci. USA 100 975-980 (2003)

14. Cross, F. Dev. Cell 4, 741-752 (2003).

\title{
Close look at gene conversion hot spots
}

\author{
Jeffrey D Wall
}

\begin{abstract}
New work has directly estimated male gene conversion rates in three regions of the human genome and identified gene conversion hot spots in the same locations as previously identified crossover hot spots. This work elucidates the fine-scale structure of linkage disequilibrium (LD) in the human genome and will be useful in association studies and other LD-based applications in population and human genetics.
\end{abstract}

Standard models suggest that there are two different forms of homologous recombination, commonly called crossover (which may be accompanied by gene conversion) and gene conversion (without an associated crossover $)^{1}$. Crossover rates vary tremendously across the human genome ${ }^{2}$, by several orders of magnitude over distances as small as $1 \mathrm{~kb}$ (ref. 3). But there are few direct data on homologous gene conversion rates ${ }^{4}$, and no information on the extent to which gene conversion rates vary across the genome. In the accompanying paper, Alec Jeffreys and Celia May ${ }^{5}$ estimated gene conversion rates at three known crossover hot spots in humans by sperm typing. They found that all three regions were gene conversion hot spots as well and that in each case, the location of the peak of conversion activity coincided with the peak of crossover rates. The coincidence of these peaks suggests that the molecular mechanisms generating most crossovers and gene conversion events are related.

\section{Recombination and LD}

Recombination is one of the primary factors that affect LD (the nonrandom association of alleles at different sites). Standard popu-

Jeffrey D. Wall is in the Program in Molecular and Computational Biology at The University of Southern California, 1042 W. 36th Place, DRB 289, Los Angeles, California 90089, USA.

e-mail: jeffwall@usc.edu lation genetics models of recombination generally ignore gene conversion, even though crossovers and gene conversions have different effects on the structure of LD. Recombination between pairs of markers that are far apart are almost exclusively crossovers, whereas pairs of markers that are close together are affected by both crossovers and gene conversion events. This is crucial for interpreting patterns of

human sequence variation. For example, analyses of human data have found less LD than expected over short distances (e.g., $<5 \mathrm{~kb}$ ) given the $\mathrm{LD}$ observed over longer distances (e.g., >100 kb; ref. 6). This seemingly discordant observation is exactly what would be expected under a model of recombination that incorporates both crossovers and gene conversion. In addition, adding gene conversion to

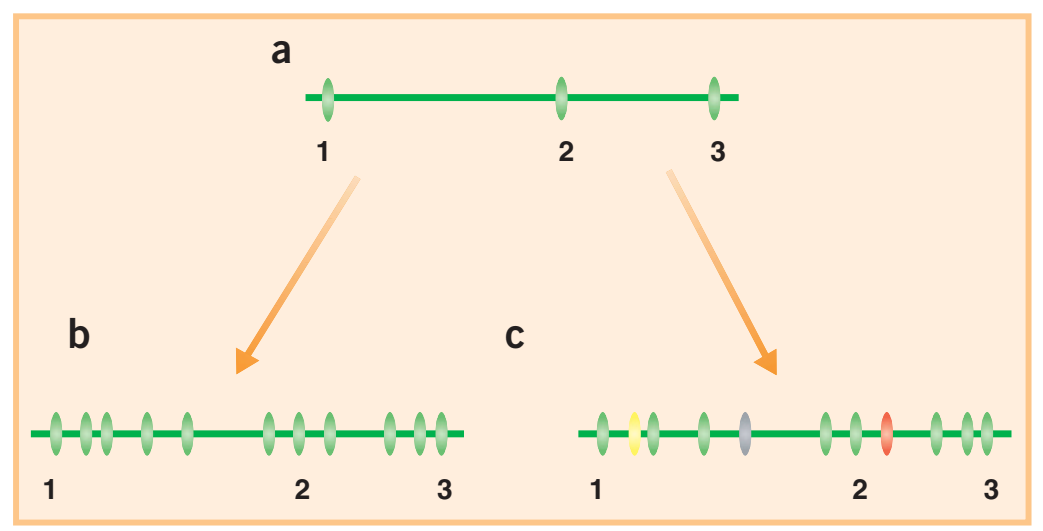

Figure 1 Schematic of the possible effects of gene conversion and marker density on patterns of LD. Horizontal lines represent chromosomes, and ovals represent markers. Ovals that are the same color are in strong LD with each other. (a) Three typed markers (labeled 1, 2 and 3) are in strong LD with each other. The patterns of LD of all the unobserved markers between these three are unknown. (b) One possibility, assumed in much of the discussion of haplotype blocks, is that all the unobserved markers are in strong LD with the end markers. (c) Another possibility, which is more probable if there are high rates of gene conversion or the original markers were far apart, is that many of the unobserved markers are in strong LD with the original three but some are not. Whether real data look more like b or $\mathbf{c}$ depends on both experimental parameters (e.g., sample size, marker density) and intrinsic parameters (e.g., gene conversion rate, crossover rate). 